\title{
Wireless, Non-invasive, Asset Life-cycle Monitoring System
}

\author{
Mark Kim ${ }^{1}$, Kevin Farinholt ${ }^{2}$, Jeff Demo ${ }^{3}$, James Eno ${ }^{4}$, Hunter Long ${ }^{5}$, Ethan Thompson ${ }^{6}$ \\ 1,2,3,4,5,6 Luna Innovations Inc., Charlottesville, VA, 22903, United States \\ kimm@lunainc.com \\ farinholtk@lunainc.com \\ demoj@lunainc.com \\ enoj@lunainc.com \\ longh@lunainc.com \\ thompsone@lunainc.com
}

\begin{abstract}
Exposure to harsh environments during storage, transportation, and handling can have significant effect on various system assets, making it important to understand the full life-cycle exposure of sensitive equipment. To quantify a system's status timeline, a wireless, intelligent, low-power life-cycle monitoring device is being developed. No current monitoring systems have the capability to measure environmental conditions experienced by an asset and use that data to determine time spent in various states such as storage, transportation, handling and deployment. The sensor system being developed will employ a multi-modal sensing approach that will provide on-device analysis of the various parameters such as acceleration, temperature, and other environmental conditions for the duration of asset storage, handling, and distribution. Extremely low power and potentially easy to integrate into existing platforms, the monitoring system will include on-system intelligence for distinguishing between different statuses and is designed to use reliable, wireless, low-profile, and inexpensive sensing technologies. Embedded system intelligence is designed to use collected datasets to accurately quantify the asset's current status and the amount of time spent within each state. The ability to monitor critical parameters and use them to classify this status throughout the asset's life-cycle could provide some of the diagnostic information that would facilitate condition based maintenance. Engineers and operators can review exposure conditions and analyze how status and exposure effects contribute to the current condition of their equipment. The monitoring system will provide maintainers additional data points in assessing the historical use of the system.
\end{abstract}

Mark Kim et al. This is an open-access article distributed under the terms of the Creative Commons Attribution 3.0 United States License, which permits unrestricted use, distribution, and reproduction in any medium, provided the original author and source are credited.

\section{INTRODUCTION}

To better understand storage and handling conditions of highvalue assets, there have been significant advances in in-situ sensors to monitor external effects throughout an equipment's life-cycle. Microelectromechanical systems (MEMS) accelerometers have been used to measure vibration and its effects on assets, but there is no sensor system available that can also track the amount of time spent within storage, transportation/handling, and in deployment. Extended exposures to varying environments during storage and transportation can impact high value electromechanical systems; therefore, it is of interest to fully comprehend the full lifecycle exposure of sensitive equipment (Ivce, R., Jurdana, I., Mohovic, R., 2011). There have been studies that track the conditions experienced by equipment for individual shipments (SenseAware, 2009) (Midè, 2016), but there currently is no solution that monitors this information throughout the full lifespan of an asset, tracking how long it has spent in differing states, both before and after shipment.

To assess status timelines and to potentially use this information to facilitate reduction in associated maintenance, an intelligent, low-power, easily retrofitted asset life-cycle status monitoring device (AssetLife) is being developed. Environmental parameters such as temperature and relative humidity could play a major role in long- and short-term aging effects of components including casings, sealants, and electronics. The AssetLife sensor system will monitor environmental factors experienced by the asset over its entire lifetime, including vibration profiles, temperature, relative humidity, light intensity, and pressure. These parameters are designed to be combined using sensor fusion algorithms to correctly quantify the time spent in storage, transportation/handling, and in operation. With this enhanced visibility into life-cycle conditions, maintainers will be able to assess equipment usage more efficiently. The developed sensing system will be extremely low power to ensure extended lifetime with limited power resources and include on-system intelligence for distinguishing between various storage or transportation conditions. The system uses 
small, reliable, inexpensive system components to enable ease of integration into existing and future platforms.

\section{BACKGROUND}

Throughout the lifetime of many fielded medical, military, and exploration systems, equipment may undergo various states of storage (indoor/outdoor/in container), handling (mechanical or human lifts), and transportation (ground or aircraft). During this time, assets are exposed to varying environmental and physical effects that can induce degradation (Muszynska, A., 1994) (Kramb, V., Hoffman, J., Johnson, J., 2003). With the emergence of cheap, readily accessible, small, low power MEMS accelerometers many wearable devices have been implemented to track human activity (Kim, Y., Kang, B., Kim, D., 2015) (Li, Z., Wei, Z., Yue, Y., Wang, H., 2011), but there is no sensor solution that is capable of predicting the activity of equipment when in states such as storage, handling and transportation. Sensors exist that monitor vibration and a limited set of environmental effects such as temperature (SenseAware, 2009) (Midè, 2016), but no sensors have been able to log time spent in various life-cycle states. To better understand the handling and storage of these systems and its long term effects on the system, it is necessary to create a non-invasive life-cycle status monitoring device to monitor various parameters experienced, and to generate a reliable status timeline.

The developed sensor node will enable a simple, inexpensive and maintenance-free tool to provide valuable insight into the conditions and their respective durations that valuable assets are exposed to throughout their life-cycle. The system provides a means for long-term monitoring of high value assets without increased labor burden and could ultimately result in improved storage and handling processes. The multi-modal sensing aspect of the proposed sensor system should provide an enhanced level of visibility to categorize and quantify times within various states.

The long term impact of this development effort will include a means for health diagnostics of a vast array of electronic and mechanical systems under exposure to a wide range of handling and ambient conditions. To provide a comprehensive solution to this problem, the sensor node must be a fully robust system capable of monitoring a suite of conditions including vibration, temperature, light, relative humidity, and barometric pressure.

\subsubsection{Vibration Effects}

Vibration and shock effects have been proven to negatively impact mechanical systems (Muszynska, A., 1994). Vibration can cause damage to electronics and enclosures which could lead to unserviceability or critical failure upon deployment.

\subsubsection{Environmental Effects}

Environmental parameters such as temperature, relative humidity, and UV exposure each play a major role in longand short-term aging effects of system components including casings, sealants, and electronics (Kramb, V., Hoffman, J., Johnson, J., 2003). High relative humidity can cause corrosion, electrolysis, and moisture absorption, introducing conductivity within insulators that reduces both mechanical and electrical reliability in certain systems. If subjected to high temperatures for extended periods of time, subsystem components could be prone to oxidation, structural changes, softening, and physical expansion. With overexposure to UV radiation, embrittlement and chemical reactions could occur, causing surface deterioration and alteration of electrical or mechanical properties. Alone, these three environmental factors could greatly affect subsystems, but when combined, deterioration of the system could be accelerated. For example, increased relative humidity or temperature could intensify the degradation effects of UV radiation on organic materials within some devices or structures.

\section{SYSTEM DESIGN}

The development of an intelligent, low-power health monitoring system carries with it a wide array of technical challenges that must be overcome to provide a suitable solution for equipment owners and maintainers. First and foremost, development of an ultra-low power system capable of continuous operation requires a unique design, optimized using the appropriate components and operational methodology. Additionally, selection and implementation of suitable sensor technologies is critical for obtaining highly accurate and repeatable measurements while maintaining ultra-low power consumption operations. Finally, efficient evaluation of operational status using embedded data and classification algorithms could provide insights into the system state and current usage of the monitored asset.

\subsection{Ultra-Low Power System Design}

Development of a micro-power monitoring system requires a design methodology that takes all components of the system into account, including the microcontroller, communications interface, long- and short-term memory, and analog interfaces. Selecting an appropriate microcontroller is the first step in designing a low power system that incorporates all of the requirements. The selected microcontroller is an ultra-low power microcontroller capable of operating at 100 $\mu \mathrm{A} / \mathrm{MHz}$ in its highest power state, allowing for much lower power consumption at lower clock speeds. Additionally, the device contains on-chip non-volatile memory, negating the need for peripheral memory components within the system. The microcontroller has been coupled with sensors to obtain measurands of interest in an early-stage system prototype (Figure 1). 


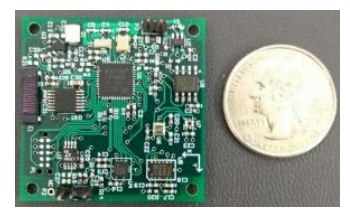

Figure 1. Initial AssetLife system prototype.

\subsection{Sensor Fusion Algorithm Measurands}

The sensors being considered for the sensing system can be categorized into three different sections: atmospheric sensors, dynamic sensors, and light intensity sensors. In each of the sensor categories, the sensors have been characterized based on power consumption, accuracy, operation range, and dimensional footprint. Other modalities such as position (GPS), inertial movement, volatile organic compounds, gas $\left(\mathrm{SO}_{2}, \mathrm{NO}_{2}\right.$, etc.) and corrosion environment sensors are currently being researched but have not yet been applied to the AssetLife sensing system.

\subsubsection{Atmospheric Sensor}

The selected environmental sensor is able to measure atmospheric conditions such as relative humidity, temperature and barometric pressure. Measurement of barometric pressure will be a key factor distinguishing between ground and air transport. Common ranges that are available from commercial sensors span from $0-100 \%(\mathrm{RH})$, -40 to $185^{\circ} \mathrm{F}$ (temperature), and 300-1,100 mbar (pressure).

\subsubsection{Dynamic Sensors}

A vibration sensor is used to provide an indication of when equipment is being handled or transported. Along with measuring vibration during normal timed intervals, the AssetLife accelerometer is responsible for "waking up" the sensing system when shock/vibration events are experienced by the asset.

\subsubsection{Light Intensity Sensors}

Two light intensity sensors were also chosen to be a part of the sensing system: a lux sensor and ultraviolet (UV) optical sensor. The lux sensor measures the intensity of visible light, while the UV sensor measures only in the 290-400 nm wavelength spectrum. The lux measurement detects ambient light, representative of what the human eye captures, helping to differentiate where an asset is stored. UV intensity will give another useful data point in differentiating whether an asset is stored indoors or outdoors.

\subsection{Embedded Intelligence}

A primary function of this system is to make a determination of the time that assets spend in various states, such as storage, handling, general transport, and in usage. The embedded intelligence is also able to track any dynamic vibration or shock events that may be experienced during transport or handling. Additionally, system intelligence must be designed to allow for optimal operations of the monitoring system itself to minimize power consumption, maximize system lifetime, and provide the most valuable information to users. The initial system prototype uses threshold values to identify critical features from the physical and environmental data that are most useful in quantifying asset status. When thresholds are exceeded in any of the measurement features, the embedded algorithm assigns a specific status label related to that feature combination. If the algorithm deems the event a change in status, the sensing system will log the measured data, time stamp, and maximum and minimum exposures for each measurement channel.

\subsubsection{Sensor Fusion Algorithms}

The sensing system will identify exposure timelines and under what environmental conditions the system operates for its entire lifetime. This status timeline data can then be given to operators and maintainers to be used to determine usage of the system. Defining usage of an asset is a complex application requiring multisensory measurements with classification algorithms to confidently and accurately define when the asset is in each of its possible storage or handling conditions. Preliminary algorithms were developed to demonstrate this concept, and rely on a decision tree structure for status classification (Figure 2).

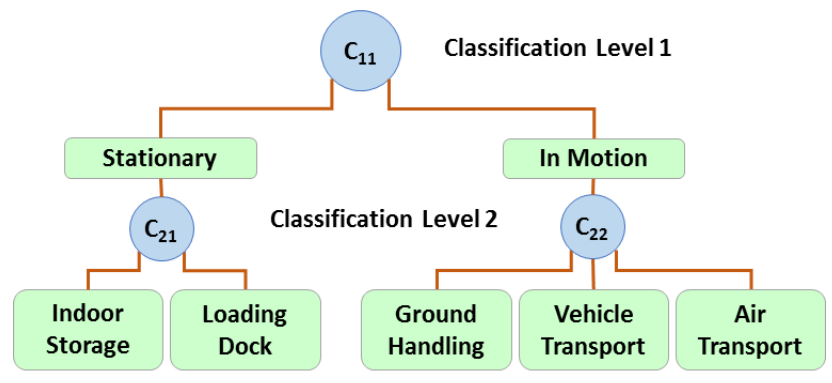

Figure 2. Preliminary structure of classification tree method to classify asset status.

Each parameter measured is categorized with thresholds, and then used as input into a decision matrix. Based on comparisons with the measurement history and current measured parameters, the embedded algorithm has sufficient information to classify the state of the system of interest. The accuracy of this approach is related to the extent of real world data collected from known states (indoor storage, loading dock, ground transport, etc.) that are used to train machine learning algorithms to establish rules within the classification method. Preliminary test data has been collected from a physical simulator and was processed using data streaming from a prototype sensing system. An extensive test regime is planned in future research and will provide data to simulate various storage, handling, and transport conditions to create a highly accurate and detailed decision matrix. 


\subsubsection{Initial Classification Algorithm}

For preliminary demonstration purposes, the initial classification algorithm uses a hierarchical approach, beginning with the barometric pressure/altitude measurement being the first criteria to pass through, followed by acceleration with a subcategory of impact or transport, and finally a last check of UV and temperature together to provide the last decision criteria. Though altitude was used in this preliminary classification algorithm, in its final implementation the algorithm will look at a combination of barometric pressure and vibration effects to differentiate ground and air transport.

The initial classification algorithm is shown graphically in Figure 3 with selection criteria represented in the blue squares and red squares denoting the possible states. The decision process begins with the topmost condition, and depending on whether the data denotes true or false, selects the path that identifies the current state of the system.

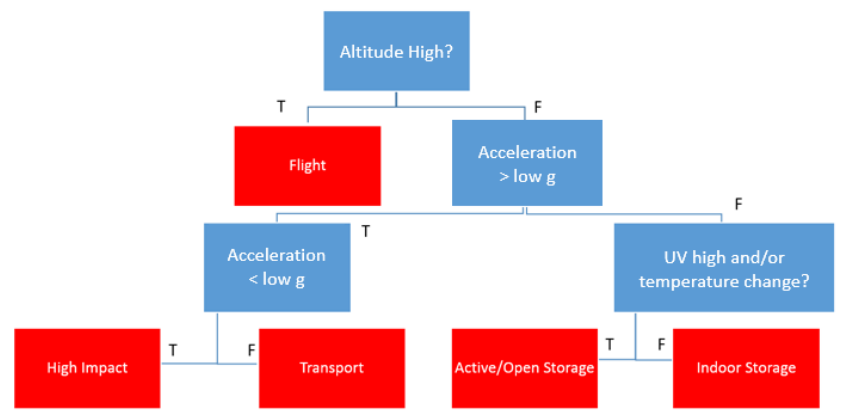

Figure 3. Decision criteria for initial testing.

\section{EXPERIMENTAL RESUltS}

After the initial sensor verification, the prototype system was attached to a test block and subjected to various conditions that an asset may be exposed to (Figure 4).

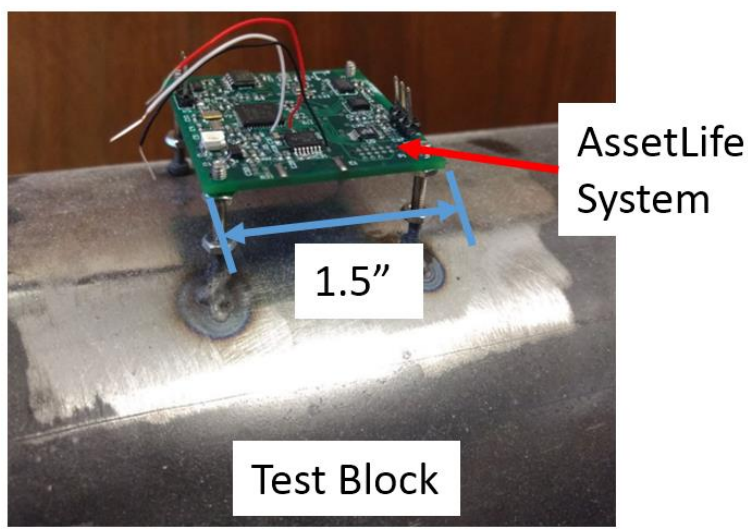

Figure 4. System mounted onto test block for simulation testing.

The sensor system hardware collected data that was then processed through initial classification algorithms executed in LabView generated code. A video camera was mounted to the test block/ as sensor data and system status were recorded (Figure 5). This video data was later used to provide a ground truth for comparison with the algorithm predictions generated throughout testing.

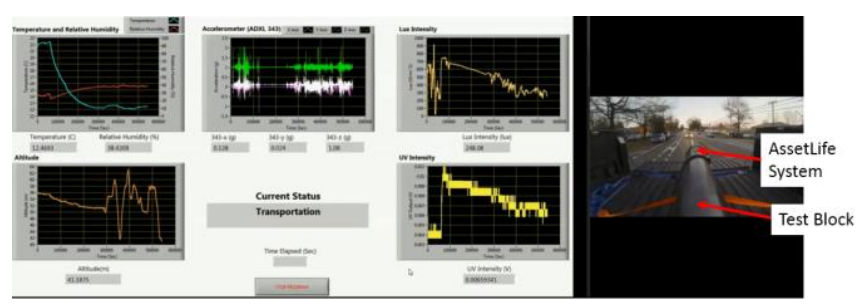

Figure 5. Visualization of real time data collected on the test block (left) and the video feed (right) used for ground truth comparison.

The full timeline of the simulated testing data collected from the sensors for this demonstration is shown in Figure 6.

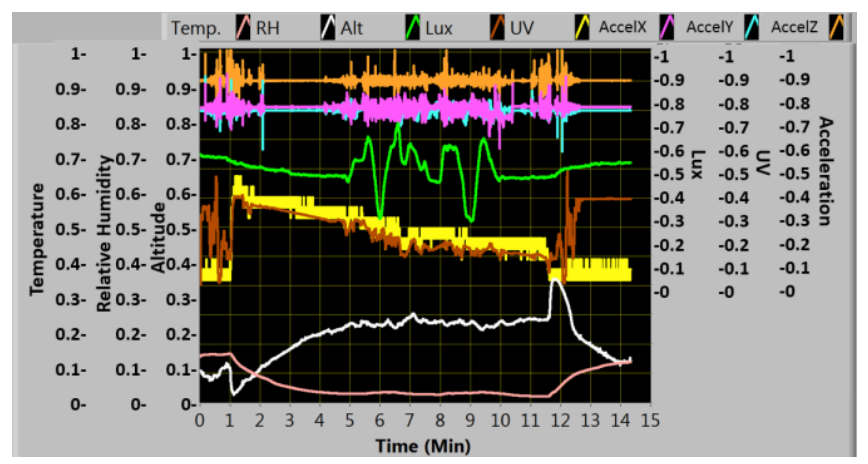

Figure 6. Complete data set of initial status classification test data.

The demonstration has been broken up into 7 phases, each showing a different status that the prototype system was able to classify. These phases are explained in detail with representative data shown in Figures 8 through 16. All data has been normalized to fit a $0-1$ scale.

Data collection began with the test block in a simulated storage environment from which it was carted outside in phase 1. During transport there is a consistent vibration response that was measured in the accelerometer output, with spikes in acceleration when the cart hits large bumps in the floor that were picked up as high impact events (Figure 7). 


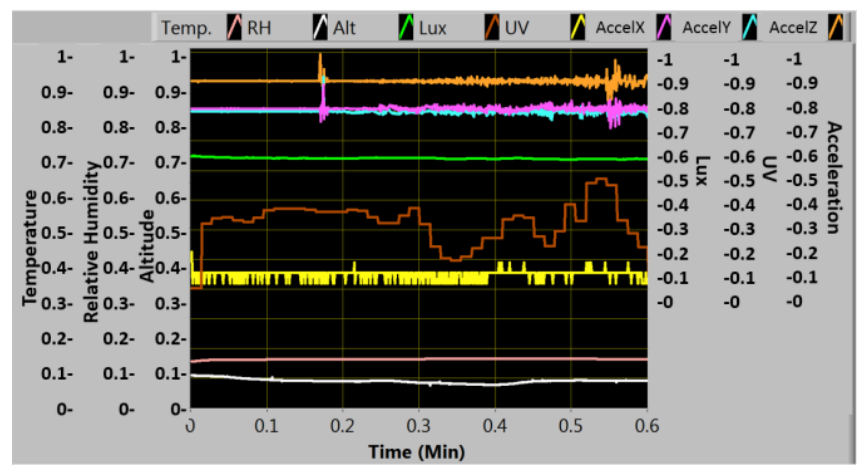

Figure 7. Phase 1 of initial testing showing high impact moment when hitting transition point in floor.

Once outside in phase 2 , the UV reading increases due to exposure to sunlight and the temperature indication decreased due to the colder outdoor temperature (Figure 8). The vibration profile during the asphalt transport is rougher than transport through the office, which is noticeable in the accelerometer data. In phase 2 of the test, the system was able to recognize this as a transportation status with the constant acceleration loading. When combined with the increase in UV level this indicates that the asset is outside.

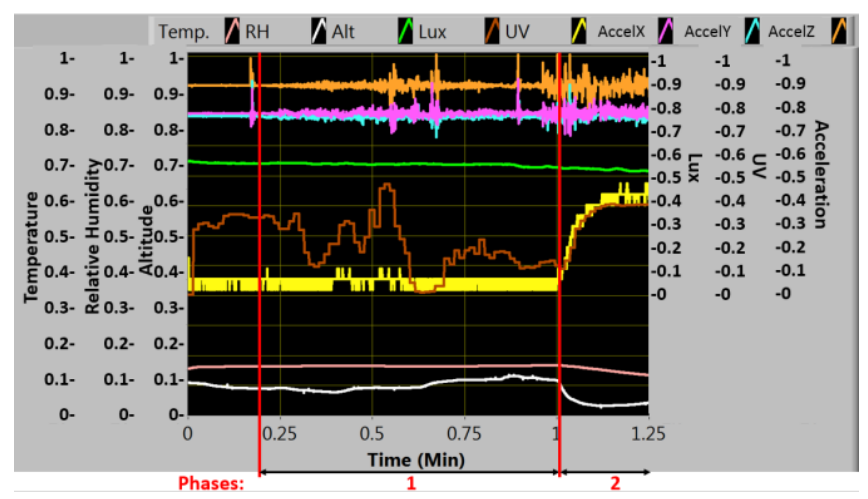

Figure 8. Phase 2 of demonstration simulating outside transportation.

In phase 3 the test block was loaded onto the bed of a transportation vehicle and secured, with the sensor system registering this as active/open air storage due to the temperature and UV change with no vibrations being picked up (Figure 9).

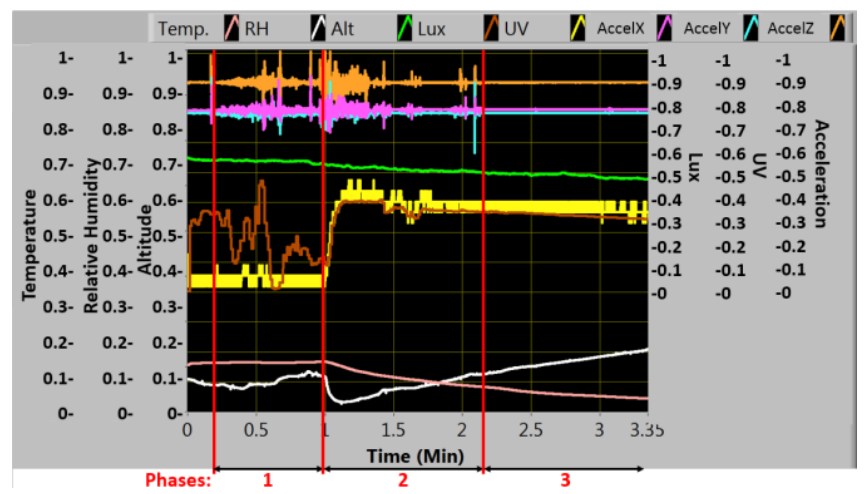

Figure 9. Phase 3 of demonstration simulating active/open storage.

Phase 4 begins as the sensing system is able to detect engine vibration from the transport vehicle as it is cranked and motion begins from transport on local roads. It can be seen that the vehicle had noticeable vibration effects, denoting the transportation state (Figure 10).

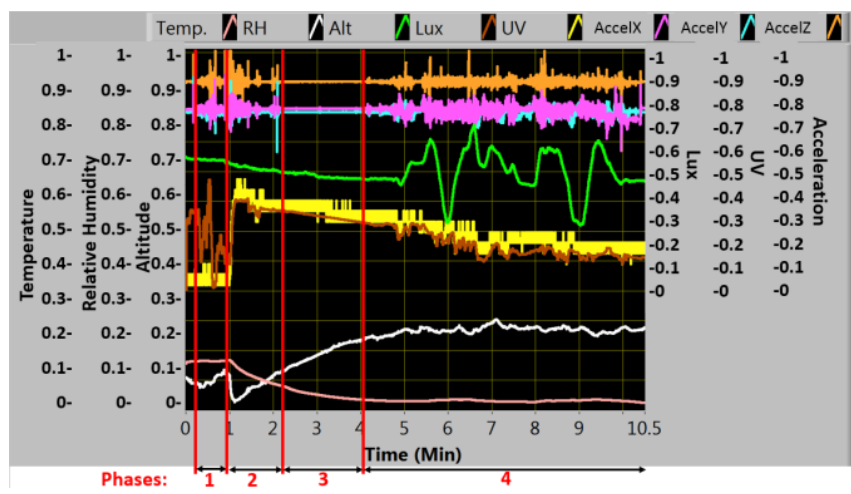

Figure 10. Phase 4 of demonstration simulating vehicular transportation of test block.

Throughout testing, the sensing system tracked barometric pressures during vehicle transport that were converted to pressure-altitudes in post-processing. Measurements were verified against the Google ${ }^{\mathrm{TM}}$ Maps altitude calculating tool (Figure 11). The Google tracking tool outputs altitude versus distance while the sensor records altitude as a function of time. Since vehicle speed varied slightly throughout this portion of testing, the altitude measurements do not match exactly, but they do provide very similar trends to verify that the system was accurately measuring and reporting barometric pressure. The Google Maps altitude output was normalized to have a similar start altitude as the asset monitoring system. 


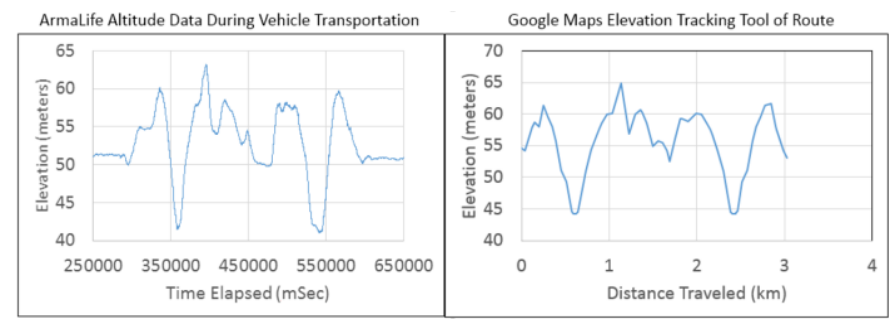

Figure 11. Verification of asset monitoring system altitude tracking abilities against an altitude route calculator from

\section{GOOGLE maps.}

After driving in phase 4, test block system was parked and all vibrations stopped, but UV was still present and the temperature was different from the storage temp, so the system was able to infer that it was in the active/open storage for phase 5 (Figure 12).

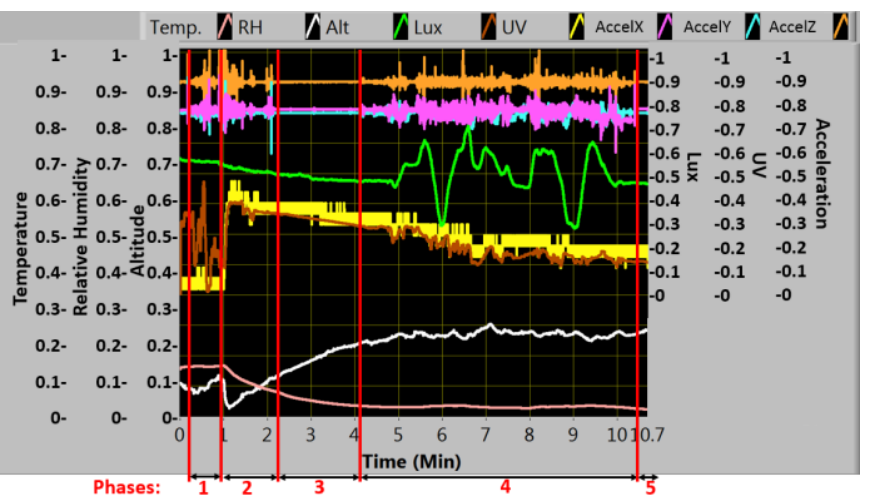

Figure 12. Phase 5 of demonstration simulating active/open storage after vehicle transportation.

In phase 6 the simulation system was loaded onto a cart and wheeled back indoors into simulated storage, where a drop in UV is noticed coincident with an increase in lux due to the overhead lighting. Temperature also begins to climb due to the warmer temperature setting indoors (Figure 13). As the test block was manually transported back into the building and into storage, the system was able to register that the test block was in transportation mode due to the acceleration response recorded by the system.

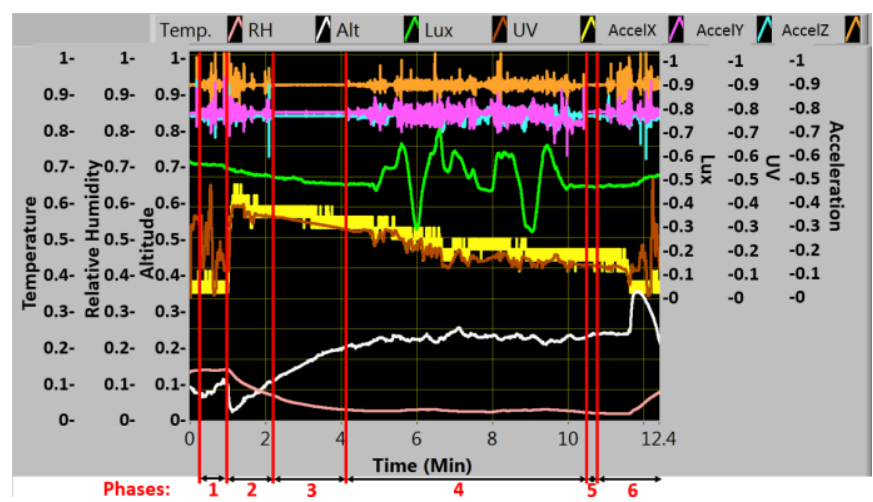

Figure 13. Phase 6 of demonstration simulating transportation back to storage.

Finally, in phase 7 the system is placed in simulated storage where the sensor readings stabilize and the sensor correctly classifies this as indoor storage (Figure 14).

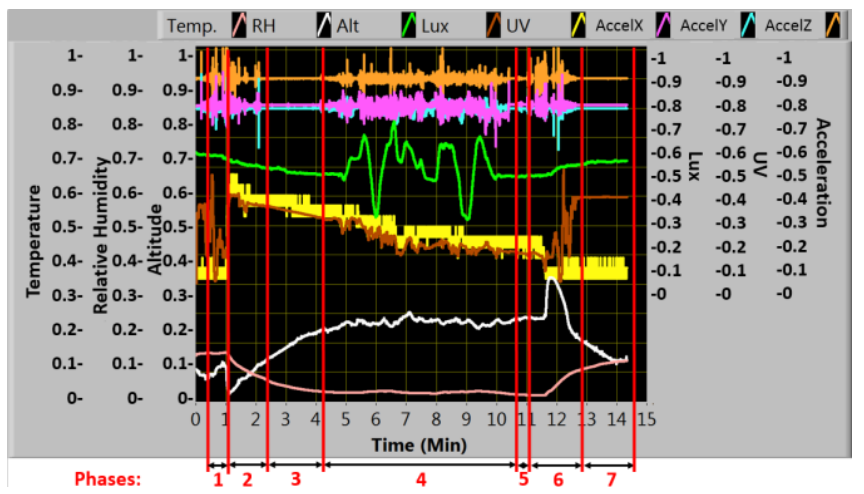

Figure 14. Phase 7 of demonstration simulating storage in office.

Using the initial classification tree algorithm presented in section 3.3.2, the AssetLife system was able to predict the status of the monitored system with $86.7 \%$ accuracy (Figure $15)$. 


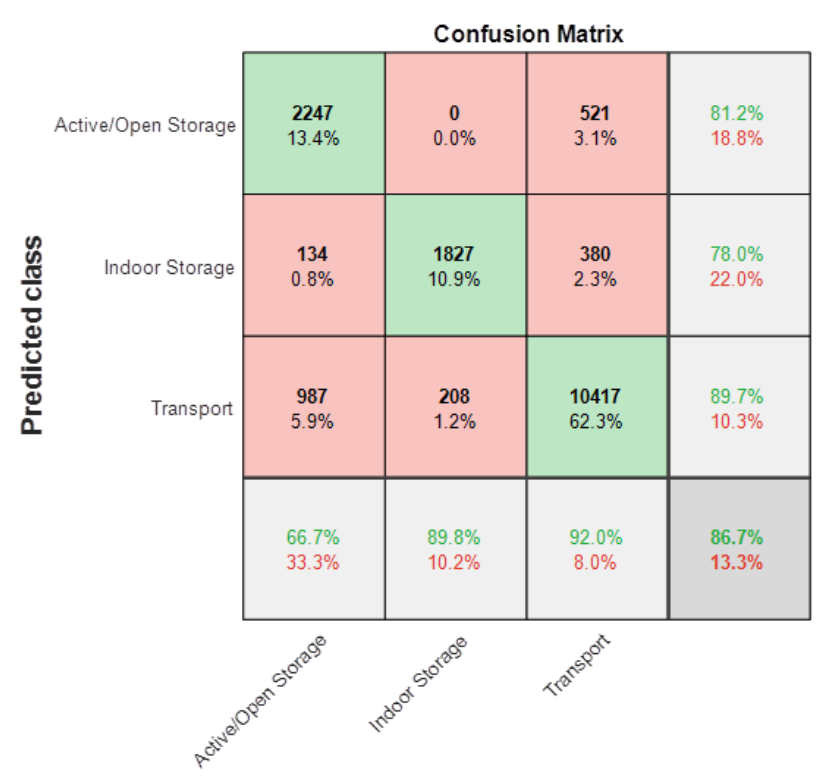

Actual class

Figure 15. Confusion matrix resulting from the initial classification tree algorithm.

The initial thresholds for the classification tree algorithm during this effort were created by performing pilot tests on the AssetLife system and manually picking thresholds from visual inspection of the test data. During the next effort of testing machine learning will be applied to improve the accuracy of the status classification algorithms.

\section{DISCUSSION AND CONCLUSION}

The preliminary AssetLife prototype is able to monitor environmental/physical effects in simulation testing, and track the amount of time spent in storage, active/open storage, and transportation. In addition to tracking the amount of time spent in various status states, the system is able to track $\mathrm{min} / \mathrm{max}$ values of asset exposure and record impact events that are experienced. The system will also be capable of storing and transferring data wirelessly to an RFID enabled data collection device.

The design approach used to develop the final asset monitoring system will be extensible to a wide range of platforms and applications. Accordingly, the hardware is designed to be low-profile and amenable to installation on different assets without interfering with normal handling procedures. Successful implementation of the sensor system will provide maintainers a diagnostic capability into storage and handling and give indications as to when and where extra precautions could be recommended to minimize variance from acceptable conditions. The AssetLife system is designed using a methodology that allows the technology to translate to a variety of high value assets, where monitoring storage, transportation, and handling could be used to track the usage of products over short or extended periods of time.

\section{ACKNOWLEDGEMENT}

This material is based upon work supported by SBIR Contract No FA8650-17-C-5056. Any opinions, findings and conclusions or recommendations expressed in this material are those of the authors and do not necessarily reflect the views of the United States Air Force.

\section{NOMENCLATURE}

FRAM Ferroelectric Random Access Memory

MEMS Microelectromechanical system

NFC Near-Field Communication

nA Nanoamps

nm Nanometers

RFID Radio Frequency Identification

RM\&A Reliability, Maintainability, and Availability

UV Ultraviolet

$\mu \mathrm{A} \quad$ Microamps

\section{REFERENCES}

Ivce, R., Jurdana, I., Mohovic, R. (2011). Vibrations monitoring on board ship with a fiber optic sensors. In ELMAR 2011 Proceedings.

Kim, Y., Kang, B., Kim, D. (2015). Hidden Markov Model Ensemble for Activity Recognition using Tri-axis Accelerometer. 2015 IEEE International Conference on Systems, Man, and Cybernetics.

Kramb, V., Hoffman, J., Johnson, J. (2003). Characterization of weathering degradation in aircraft polymeric coatings using NDE imaging techniques.

Li, Z., Wei, Z., Yue, Y., Wang, H. (2011). An Adaptive Hidden Markov Model for Activity Recognition Based on a Wearable Multi-Sensor Device. J Med Syst (2015)

Midè, What Really Happens to Your Package During Shipment?. https://blog.mide.com/transportationvibration-monitoring-what-really-happens-duringshipment

Muszynska, A. (1994). Vibrational Diagnostics of Rotating Machinery Malfunctions. International Journal of Rotating Machinery, Volume 1, Issue 3-4, Pages 237266.

SenseAware. (2009) Monitor Your Shipments With SenseAware ${ }^{\circledR} \quad$ Powered by FedEx. https://smallbusiness.fedex.com/dec-sensaware.html

\section{BIOGRAPHIES}

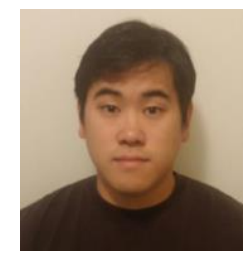

Mark Kim received his Bachelor's degree in Electrical Engineering from Virginia Polytechnic Institute and State University in Blacksburg, VA. His first career was at Matsys Inc. located in Sterling, VA, a materials and systems engineering company. He was the lead Electrical engineer and was in charge of programming, circuit design and implementation, operation and analysis of the 
high temperature $(>1100 \mathrm{C})$ Eddy Current sensors, and electrical systems lead on multiple SBIR programs. He oversaw multiple field tests of complex multi-modal metal composite materials and performed analysis on data collected. While at Matsys he developed a novel inexpensive Delubrication monitoring device to be used in the commercial application of sintering. Mark joined Luna in 2015 as an electrical engineer and is currently designing compact, low-power, embedded electrical system hardware. Mark is a member of IEEE and the IEEE Aerospace and Electronics Systems Society.

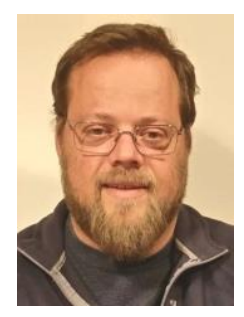

Kevin Farinholt is a Senior Research Engineer with the Performance Systems and Analytics Group at Luna Innovations. His research focuses on equipment and structural health monitoring for high value systems. He received his B.S., M.S., and $\mathrm{Ph} . \mathrm{D}$. in mechanical engineering from Virginia Polytechnic Institute and State University in Blacksburg, VA. He has worked in active material development, modeling, and characterization, embedded sensing systems, and health monitoring for several research laboratories, including Los Alamos National Laboratory and the Commonwealth Center for Advanced Manufacturing. He has chaired conferences on Industrial and Commercial Applications of Smart Structures Technologies, and is currently a member of the ASME Adaptive Structures and Material Systems Technical Branch

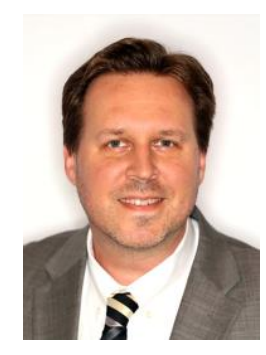

Jeff Demo is the Electrical Systems Team Lead in the Performance Systems and Analytics Group at Luna Innovations. He has led multiple SBIR and non-SBIR programs related asset health monitoring and condition based maintenance. His primary focus while at Luna has been sensor development, instrumentation, data collection, and embedded system design, specifically associated with Luna's ultra-low power wireless sensing platform. Prior to working with Luna, Jeff developed aerospace electronics subsystems, including work on the F-35 Joint Strike Fighter's air data system. Jeff is a senior member of IEEE and the IEEE Aerospace and Electronics Systems Society.

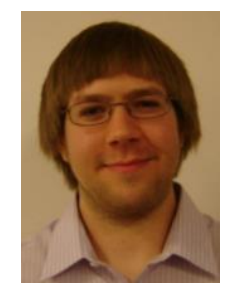

Ethan Thompson received his Bachelor's degree in Engineering with a minor in Computer Science from James Madison University in Harrisonburg, VA. At JMU, Ethan led the embedded hardware development of a GPS tracking device that communicated its location data to a base station real-time via wireless Cellular communication. This project was aimed at collecting location data on Matatu busses in Nairobi, Kenya, with the goal of helping city planners design smarter bus routes to reduce pollution and traffic congestion throughout Nairobi. Ethan began his career at Luna Innovations in 2014 as a Firmware Engineer. Ethan has taken the lead firmware development role in numerous projects at Luna. In his time at Luna, he has gained in-depth experience with Texas Instruments' Ultra-Low Power MSP430 and MSP432 microcontrollers that are used in projects related to condition based maintenance evaluation of various systems and structures. Ethan is a member of the IEEE and the IEEE Systems, Man, and Cybernetics society.

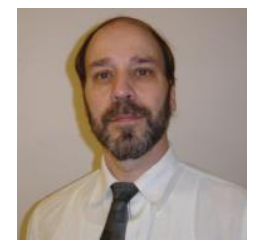

James Eno received his Bachelor of Applied Science (BASc) degree in Electrical Engineering from the University of Waterloo in Ontario. His initial employment with Philips Electronics, Inc. located in Toronto involved the development of linear and digital video circuitry for the commercial TV market. Jim found opportunities with Harris Corporation where he wrote embedded firmware for base station repeaters and portable/mobile radio products for Public Safety communications. He enriched the feature set and reliability of radio communications meeting the high demand of Police, Fire and Ambulance services worldwide. He also developed the hardware, firmware and PLD logic for an Adaptive Differential Pulse Code Modulation (ADPCM) codec to support the coupling of legacy analog base stations with the system's digital infrastructure. Jim joined Luna in 2016 as an electrical engineer and is actively designing embedded firmware to drive a variety of sophisticated health monitoring technologies.

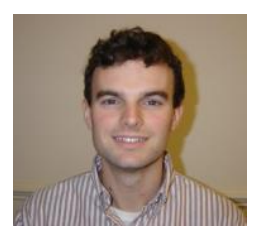

Hunter Long received his Bachelor's and Master's degrees in Electrical Engineering from Virginia Tech in Blacksburg, VA. As part of his Master's degree, Hunter researched fast-scan cyclic voltammetry and Raman spectroscopy and their applications to measuring concentrations of neurotransmitters in the human brain. He also investigated different machine learning techniques and their applications to neuroimaging. Before coming to Luna Hunter worked for the Naval Research Lab in Washington D.C. and Areva NP in Lynchburg, VA. At the Naval Research Lab Hunter was a member of the Freespace Photonic Communications office where he helped conduct research on novel optical systems. At Areva NP he was responsible for designing analog and embedded circuitry, writing firmware, and testing nuclear safety related devices. Hunter joined Luna in 2016 as an electrical engineer and is currently designing compact, low-power, embedded electrical system hardware. 